\title{
e-Phaïstos
}

e-Phaïstos

Revue d'histoire des techniques / Journal of the history

of technology

III-1 | 2014

Varia

\section{Voir et concevoir : les théâtres de machines (XVIe- XVIIIe siècle)}

Seeing and designing: theaters of machines (16th-18th century)

\section{Benjamin Ravier-Mazzocco}

\section{(2) OpenEdition}

Journals

Édition électronique

URL : http://journals.openedition.org/ephaistos/648

DOI : $10.4000 /$ ephaistos. 648

ISSN : 2552-0741

Éditeur

IHMC - Institut d'histoire moderne et contemporaine (UMR 8066)

Édition imprimée

Date de publication : 1 juin 2014

Pagination : 100-104

ISSN : 2262-7340

\section{Référence électronique}

Benjamin Ravier-Mazzocco, « Voir et concevoir : les théâtres de machines (XVle-XVIIle siècle) 》, e-

Phaïstos [En ligne], III-1 | 2014, mis en ligne le 24 novembre 2016, consulté le 10 décembre 2020. URL : http://journals.openedition.org/ephaistos/648 ; DOI : https://doi.org/10.4000/ephaistos.648 


\title{
Benjamin Ravier-Mazzocco
}

\section{Voir et concevoir : les théâtres de machines (XVIe-XVIIIe siècle)}

\author{
Thèse de doctorat \\ Université Paris 1 Panthéon-Sorbonne \\ Centre d'histoire des techniques (CH2ST/EA127) \\ Soutenue le 12 septembre 2013 \\ Un volume de texte(494 p.), un volume d'annexes (544 p).
}

Directeur de thèse :

Anne-Françoise GARÇON, professeur, Université Paris 1 Panthéon-Sorbonne

Jury:

Pascal BRIOIST, professeur, Université François Rabelais, Tours

Jean-Luc Chappey, maître de conférences habilité à diriger des recherches, Université Paris 1

Pascal DUBOURG-GLATIGNY, chargé de recherche, Centre Alexandre Koyré, CNRS

Hélène VERIN, chargée de recherche Centre Alexandre Koyré, CNRS

Jakob VOGEL, professeur, Centre d'histoire de Sciences Po, FNSP
Monsieur le Président,

Mesdames et Messieurs, membres du jury,

Je suis très honoré de pouvoir présenter devant vous cette thèse de doctorat d'histoire intitulée: Voir et concevoir : les théâtres de machines (XVI ${ }^{e_{-}}$ $X V I I I^{e}$ siècle). Au cœur de la problématique se trouve l'histoire d'une pensée technique particulière : celle des ingénieurs, ces hommes de l'entre deux, entre science et pratique, entre commanditaire et ouvrier. Cette interrogation est d'abord le fruit d'une histoire personnelle marquée par un entourage d'ingénieurs : un grand-père qui dessinait des alternateurs de barrages hydrauliques pour Alstom, un père qui travaille à la qualité du combustible nucléaire chez Areva, deux frères aînés ingénieurs informaticiens. Autant dire que j'étais familiarisé avec la pensée des ingénieurs contemporains. Quoi de plus attractif alors qu'un sujet qui me permettait d'envisager cette pensée sous un angle historique? Cet axe de recherche cependant n'allait pas de soi et fut long à construire.

Pour mon entrée en master à l'ENS de Lyon en 2007, je cherchais à m'orienter en histoire des sciences de la Renaissance. Olivier Christin, alors professeur de l'Université Lyon 2, accepta de diriger mon travail et me proposa deux sujets: l'un sur l'académie des sciences de Lyon, et l'autre sur les théâtres de machines. J'avais un mois pour choisir. 
J'allais voir de quoi il s'agissait au fond ancien de la bibliothèque de Lyon (les collections étaient peu numérisées). Je choisis alors sans hésiter les théâtres de machines.

Cette rencontre avec les sources fut fondamentale pour toute ma recherche. Outre leur esthétique soignée, ce qui m'attirait était un hiatus entre le sérieux dont le texte se parait et le faste des images. Cet écart faisait écho à la Fabrica de Vésale que j'avais étudié en licence, mais la comparaison n'épuisait pas le sens de ces livres. Il me semblait y avoir quelque chose de plus "transgressif » dans cette multiplication de dispositifs mécaniques. Ces livres étaient très "renaissants ", ils avaient une étrangeté qui les rendait difficiles à comprendre pour notre vision contemporaine des techniques et des machines. Ma première année porta sur l'histoire des éditions de Jacques Besson. Et la soutenance eut lieu au centre Malher, Olivier Christin souhaitant qu'Anne-Françoise Garçon fût présente. Il fût alors élu à la présidence de l'université Lyon 2, j'entamais donc ma deuxième année de master sous la direction de Mme Garçon.

Le fait d'être entré dans cette recherche par les sources était un défi à ma formation d'historien. La plupart de mes camarades (à l'ENS comme à l'université Paris 1) fondaient leur recherche sur un raisonnement inverse: ils partaient d'une interrogation première et choisissaient, moissonnaient les sources en fonction de cet axe de recherche. Quant à moi, j'étais confronté à une multitude de pistes ouvertes par une historiographie abondante: l'évolution du graphisme technique avec Yves Deforges ; le rôle de ces ouvrages dans l'imaginaire et l'art européen avec Alexander Keller et Samuel Edgerton ; les liens avec les manuscrits médiévaux après Ladislao Reti ; les liens avec la Réforme et le marché des curiosités avec Luisa Dolza ; l'analyse de la pensée technique des ingénieurs avec Hélène Vérin.

Il était nécessaire de recentrer le sujet sur des axes clairs, de choisir un angle d'approche de ces sources à première vue déroutantes. Dans ces premières années, deux questions me revenaient régulièrement qui me furent difficile à verbaliser. La première était de comprendre quelle pensée présidait à la rédaction de ces ouvrages. La seconde visait à saisir la logique justifiant la diversité non seulement des machines mais aussi des choix éditoriaux. Au fur et à mesure des séminaires et des discussions avec ma directrice et les membres du laboratoire, il sembla que répondre à ces questions passait par trois axes principaux.

D’abord, il fallait mieux caractériser la réception des théâtres de machines pour comprendre l'origine de leur succès. Il s'agissait d'étudier les lecteurs de ces livres sur le long terme (deux siècles), et de déterminer, dans la mesure du possible les différents types de lectures auxquels ces ouvrages avaient donné lieu. La tâche était ardue. Non seulement le corpus offrait des contenus très divers, mais les usages et les lectures de ces ouvrages furent aussi multiformes. Je décidais de réaliser d'abord une étude quantitative permettant d'avoir une idée de la diffusion des théâtres de machines en Europe. Je complétais ensuite cette première étude par multitude d'études de cas afin de rendre compte de la diversité des lectures possibles, tant parmi les ingénieurs, que les bibliophiles, les savants ou les pédagogues. Et il apparut alors clairement qu'une des clés du succès durable de ce genre particulier (certains titres célèbres sont encore cités au début du XIX ${ }^{\mathrm{e}}$ siècle), tenait à la pluralité des usages et des lectures.

Cette étude de la réception m'a amené - second axe- à étudier plus en détail les théâtres de machines du XVIIIe siècle, qui n'avaient fait l'objet que d'assez peu d'études, et qui étaient régulièrement placés en dehors du genre des "vrais " théâtres de machines de la fin de la Renaissance. Pourtant, dans leur titre même, ils se posaient en héritiers de cette ancienne tradition, et une analyse plus fine révéla en quoi ils formaient davantage une évolution des théâtres de machines que l'émergence d'un nouveau type d'ouvrages. Dans ce cadre, les Theatri Machinarum de Jacob 
Leupold m'ont semblé beaucoup plus intéressant, car relevant d'une démarche nouvelle et explicitée ; aussi je choisissais de l'étudier plus en profondeur. Les sept tomes étaient écrits dans un allemand ancien, ponctué d'expressions techniques. Il n'a fait l'objet que d'une seule traduction en anglais, dans un manuscrit inédit daté du XVIII e siècle, écrite sans doute en partie par Charles Hamilton, et actuellement conservée à la Huntigton Library, en Californie. Georges Wilfried Lockett travailla d'ailleurs sur cette traduction manuscrite, tout en notant son caractère "inégal » (« uneven »). Pour ma part, je n'avais pas la chance de pouvoir consulter la moindre traduction. Il me fallut lire l'ouvrage dans sa langue originale, et en traduire de larges passages. Un effort qui nécessita un temps conséquent, mais qui s'avéra payant. En effet, Jacob Leupold formalisait ici un raisonnement de conception demeuré largement dans l'implicite avant lui. Il ouvrait ainsi la voie à d'autres ouvrages technologiques du XIX ${ }^{\mathrm{e}}$ siècle comme celui de G-A. Borgnis.

En retour, il devenait nécessaire de mieux saisir l'intérêt technique des premiers théâtres de machines : ce qui s'offrait de façon directe et claire chez Leupold était beaucoup plus difficile à démêler dans les premiers ouvrages. Aussi, sur ce dernier point, de loin le plus difficile à conceptualiser, deux rencontres me furent d'une aide précieuse.

L'une avec le centre Malher d'histoire des techniques, ses séminaires et ses étudiants aux sujets si divers. Un ensemble qui reposait sur une assise théorique solide en termes de pensée et de culture technique. Trois concepts développés me furent particulièrement précieux : les régimes de la pensée opératoire (pratique, technique, technologique) ; la notion si riche de "moment », qui permettait d'expliquer les similarités avec d'autres périodes sans pour autant présupposer de lois historiques ou nécessiter la mise en place d'un modèle diffusionniste; et enfin le concept de topique, qui permettait d'analyser la dynamique de pensée d'un groupe professionnel sur un objet à partir de « lieux », c'est-à-dire de caractéristiques particulières à cet objet. Enfin, en sus de ces concepts traités, Mme Garçon me demanda de travailler sur un projet d'histoire de la conception. Cette piste que j'explorais avec intérêt semblait très prometteuse pour comprendre le fonctionnement interne des théâtres de machines, et montrer leur importance dans une histoire intellectuelle des ingénieurs, une hypothèse qui ne fit que se confirmer par la suite.

Concernant l'histoire intellectuelle des ingénieurs (et la formalisation de leurs connaissances techniques), je savais ne pas pouvoir passer à côté des travaux d'Hélène Vérin. Ce fut la seconde rencontre. En réalité, ses textes furent parmi les premiers que j'avais lus, mais je n'en comprenais pas toute la portée. Il me fallait d'abord acquérir une meilleure familiarité avec les sources pour comprendre ce qui était mis en mots : le rôle légitimant des théâtres de machines, l'importance qu'y prenaient la cinématique et les combinaisons, la place des ouvrages dans une histoire bien comprise de l'invention. Je souhaitais alors préciser les choses, poursuivre des axes proposés, compléter cette base, notamment à l'aide d'une étude technique plus poussée et de données quantitatives.

Cette exigence me conduisit à la réalisation d'une base de données. Le travail était à peine initié que je découvrais celle de Markus Popplow et Georges Lefebvre. Elle présentait l'avantage d'être disponible et d'offrir des outils efficaces pour analyser le graphisme et les légendes du corpus dans une perspective comparative. Par contre, si elle listait les dispositifs mécaniques présents dans les machines, elle présentait l'inconvénient de ne pas autoriser la prise en compte des relations cinématiques dans les machines. C'est pourquoi il me fallait opérer dans ce sens. Une exigence qui demandait un haut degré de technicité dans la conception de la base de données, et nécessita des compétences supérieures à celles dispensées aux historiens, et que je trouvai chez mon frère informaticien. 
Mais plus que sur le défi technique, j'aimerais insister sur le défi intellectuel que représentait cette base, qui fut au moins aussi grand. En effet : quand on voit des résultats statistiques, on les qualifie toujours de " quantitatifs », oubliant que c'est aussi un processus «qualitatif ». La construction de la base de données est au moins aussi importante que les résultats qu'elle donne. J'insiste beaucoup sur ce point. Pour réaliser une structure cohérente, il faut faire des choix, définir un angle d'analyse et le développer, avec rigueur, jusque dans ses détails, même si ceux-ci sont à première vue insignifiants. Le choix des catégories doit être clair et ne supporte pas le flou. Le choix de rejeter certaines précisions doit être clairement énoncé. Ainsi, même si cela peut paraitre inattendu, construire ma base de données fut la meilleure occasion de poser toutes mes hypothèses, de débusquer tous mes préjugés, de clarifier tous mes choix.

$\mathrm{Au}$ final, quels furent les résultats principaux de ce travail ? J'en distinguerais quatre.

Premièrement, l'évolution du genre, qu'il est possible de diviser en quatre phases. La première est celle des livres d'inventions, de 1570 à 1587 . Ici, les auteurs, ingénieurs de métier, cherchaient à mettre en avant leur ingenium, leur génie mécanique. Il s'agissait à la fois de se mettre en valeur et de séduire de probables commanditaires. De ce fait même, les légendes étaient courtes et donnaient peu d'explications techniques et le raisonnement de conception était entièrement implicite. En revanche, le discours de légitimation, individuel et collectif, prenait ses premières marques. La seconde phase est celle des théâtres de machines proprement dits, de 1578 à 1629. Les éditions genevoises du Besson et la publication du Ramelli sont ici un tournant. Les auteurs sont moins des ingénieurs que des éditeurs humanistes ou des amateurs éclairés. Nous voyons se mettre en œuvre une pédagogie, issue d'une volonté d'expliquer le fonctionnement des machines: les légendes augmentent en taille, dévoilant la cinématique des machines ; les ouvrages voient leur organisation petit à petit explicitée jusqu'à transformer le genre en une série de catalogues. La troisième phase est celle des encyclopédies de machines qui se développent dans l'espace technique de langue allemande, à partir de 1607 et pendant tout le XVII ${ }^{\mathrm{e}}$ siècle. Porté par des éditeurs germaniques, il s'agit de regrouper toutes les machines, de les décrire, d'en préciser les caractéristiques (matériaux, taille, etc), et de les intégrer dans le cadre d'une science statique récemment formalisée. Le cas des Theatri machinarum d'Heinrich Zeising est ici symptomatique de cette évolution. Enfin, héritier principal de cette dernière tradition, le genre mue à partir de 1727 vers la technologie mécanique, à travers notamment la publication des Theatri machinarum de Jacob Leupold. Il s'agit explicitement de former une classe intermédiaire entre science et pratique, capable d'administrer la technique, à l'aide de principes issus de la confrontation entre une science statique dont les limites commencent à se faire sentir et une expérience pratique des machines. De justification, l'objectif est devenu un objectif de formation des ingénieurs.

Deuxièmement, l'étude technique des ouvrages permit de préciser le raisonnement de conception à l'œuvre dans les théâtres de machines. La notion de topique telle qu'elle est développée au laboratoire fut ici très importante pour expliquer ces résultats et préciser les différentes modalités possibles de la conception. Elle exprime bien la dynamique qui permettait aux auteurs de trouver la cinématique efficace pour résoudre un problème à partir de séries fonctionnelles (par exemple " élever l'eau »), et du déploiement de lieu par la multiplication des combinaisons (comme dans le cas de Ramelli), ou par un jeu de variation sur les "principes fondamentaux », les «machines simples » (comme dans le cas de Besson).

Troisièmement, les études sur la réception du genre ont mis en évidence les usages différenciés des théâtres de machines. Objets de collections 
curieux et rares pour des bibliophiles avertis; ils furent sans doute utilisés aussi comme des catalogues d'inventions chez plusieurs amateurs. Dès la fin du XVII e siècle, il devient évident qu'ils complétaient aussi un enseignement de mécanique en servant de bibliothèque de situations; tout comme ils influencèrent enfin la constitution de certaines collections de modèles et la rédaction de certains catalogues. Cela se traduit par une concentration des ouvrages dans les bibliothèques d'établissements spécialisés dans l'étude des mathématiques «mixtes" (certains collèges jésuites, puis écoles d'ingénieurs).

Quatrièmement, la place de l'œuvre de Jacob Leupold est mieux connue. Il symbolise la quatrième phase de l'évolution du genre, qui formalise définitivement un raisonnement à la fois de conception et d'expertise, fondé sur des principes renouvelés par l'apport de la science statique et d'un nouvel usage de l'expérimentation. Cela en fait un ouvrage technologique, qui ouvre la voie des grands cours de machines du XIX ${ }^{\mathrm{e}}$ siècle, et notamment à Borgnis, qui, taisant ce qu'il doit à cette tradition antérieure, la dépasse en l'insérant dans un programme technologique de plus grande ampleur encore.

Pour conclure, j'aimerais imaginer avec vous ce que pourrait être les grandes lignes d'un nouveau programme de recherche sur le sujet. Je vois notamment deux axes majeurs de développement. Le premier serait de développer l'étude de la réception. De nombreux indices suggèrent en effet que le corpus intéressa des catégories professionnelles comme les architectes ou les maitres de l'industrie, qui furent difficiles à repérer quantitativement dans mes recherches. Il faudrait aussi creuser l'influence de cette littérature sur la pensée technique de la Nouvelle-Espagne et de la Russie. Le second axe serait de confronter ces résultats sur la pensée technique à la pratique effective des ingénieurs. Comment cela s'est-il traduit? Si on sait l'importance grandissante de la mathématisation des problèmes, l'impact des topiques inventives reste à préciser. Y a-t-il eu plus d'inventions? Plus de vocations d'inventeurs? Observe-t-on un impact des théâtres de machines sur l'évolution technique du XVII e siècle ? Plusieurs indices, notamment dans les machines proposées à l'Académie Royale des Sciences de Paris, laissent penser qu'au moins de façon marginale, cet impact a existé. Reste à savoir ce qu'il en est à plus grande échelle.

Monsieur le président, Mesdames et Messieurs les membres du jury, je vous remercie de votre attention. 